\title{
Caracterização de pacientes intoxicados por drogas de abuso em terapia intensiva
}

\author{
Cleiton José Santana ${ }^{1,2}$ \\ (iD) https://orcid.org/0000-0002-8150-2357 \\ Anai Adario Hungaro ${ }^{1,3}$ \\ (D) https://orcid.org/0000-0002-0914-5308 \\ Rosangela Cristophoro ${ }^{1,4}$ \\ (iD) https://orcid.org/0000-0002-5813-201X \\ Indianathan de Kassia Santana Elvira ${ }^{1,5}$ \\ (D) https://orcid.org/0000-0003-4827-1523 \\ Aroldo Gavioli1,6 \\ (iD) https://orcid.org/0000-0003-1454-1652 \\ Magda Lúcia Félix de Oliveira ${ }^{1,4}$ \\ (iD) https://orcid.org/0000-0003-4095-9382
}

Universidade Estadual de Maringá, Maringá, PR, Brasil.

2 Secretaria Municipal de Saúde, Diretoria de Urgência e Emergência em Saúde, Londrina, PR, Brasil.

3 Universidade Estadual de Maringá, Centro de Controle de Intoxicações, Maringá, PR, Brasil.

${ }^{4}$ Universidade Estadual de Maringá, Departamento de Enfermagem, Maringá, PR, Brasil.

5 Secretaria Municipal de Saúde, Serviço de Atendimento Móvel de Urgência, Maringá, PR, Brasil.

${ }^{6}$ Hospital Universitário Regional de Maringá, Maringá, PR, Brasil.
Objetivo: caracterizar o perfil epidemiológico de internações em terapia intensiva decorrentes de efeitos secundários do uso de álcool e outras drogas de abuso. Método: estudo transversal de 138 fichas de internados em terapia intensiva adulto de hospital escola com efeitos secundários à intoxicação por drogas de abuso. Utilizaram-se estatísticas descritivas e univariada. Resultados: a média anual de internações foi $27,6 /$ mês, com maioria do sexo masculino $(89,1 \%)$, idade média de 47,9 anos, usuários crônicos de álcool $(92,75 \%)$ e com doença orgânica secundária ao uso de drogas $(66,64 \%)$. O tempo médio de internação foi de 16,6 dias e 38,4\% evoluíram a óbito. Conclusão: neste estudo, prevaleceram internados em estado crítico do sexo masculino, em idade economicamente ativa, por uso crônico de álcool e longo período de internação. Os óbitos foram estatisticamente associados à idade maior que 40 anos, a doenças do sistema digestivo e internações com duração até 17 dias.

Descritores: Enfermagem; Hospitalização; Unidades de Cuidados Intensivos; Alcoolismo; Epidemiologia.

\section{Como citar este artigo}

Santana CJ, Hungaro AA, Cristophoro R, Elvira IKS, Gavioli A, Oliveira MLF. Characterization of patients intoxicated by drug use in intensive care. SMAD, Rev Eletrônica Saúde Mental Álcool Drog. 2020;16(1):1-8. doi: https://dx.doi.org/10.11606/issn.1806-6976.smad.2020.151960 


\section{Characterization of patients intoxicated by drug use in intensive care}

Objective: to characterize the epidemiological profile of intensive care hospitalizations due to the side effects of alcohol use and other drugs. Method: a cross-sectional study of 138 hospital admission in an adult intensive care unit of a teaching hospital, by side effects to abuse of drug intoxication. Descriptive and univariate statistics were used. Results: the mean annual hospitalizations were $27.6 /$ month, and the majority of males (89.1\%), mean age of 47.9 years, chronic alcohol users $(92.75 \%)$ and with organic disease secondary to drugs use $(66.64 \%)$. The mean length of hospital stay was 16.6 days, and 38.4\% died. Conclusion: in this study, critically ill male patients, at an economically active age, were subjected to chronic alcohol use and a long hospitalization period. Deaths were statistically associated with age greater than 40 years, diseases of the digestive system and hospitalizations lasting up to 17 days.

Descriptors: Nursing; Hospitalization; Intensive Care Units; Alcoholism; Epidemiology.

\section{Caracterización de pacientes intoxicados por drogas de abuso en terapia intensiva}

Objetivo: caracterizar el perfil epidemiológico de hospitalizaciones por cuidados intensivos debido a los efectos secundarios del consumo de alcohol y otras drogas. Método: estudio transversal de 138 registros hospitalarios en una unidad de cuidados intensivos para adultos de un hospital docente, em consecuencia de efectos del abuso de drogas. Se utilizó estadística descriptiva y univariada. Resultados: el promedio anual de hospitalizaciones fue de 27.6/mes y la mayoría era de hombres (89.1\%), edad promedio de 47.9 años, consumidores crónicos de alcohol $(92.75 \%)$ y con enfermedad orgánica secundaria al uso de drogas (66.64\%). La duración media de la estancia hospitalaria fue de 16,6 días y el 38,4\% falleció. Conclusión: en este estudio, prevalecieron internados en estado crítico del sexo masculino, en edad económicamente activa, por uso crónico de alcohol y largo período de internación. Las muertes fueron estadísticamente asociadas a edad mayor que 40 años, enfermedades del sistema digestivo y en internaciones con duración hasta 17 días.

Descriptores: Enfermería; Hospitalización; Unidades de Cuidados Intensivos; Alcoholismo; Epidemiología. 


\section{Introdução}

O uso abusivo de drogas é uma prática humana, milenar e universal, não existindo sociedade que não tenha recorrido ao seu uso com as finalidades mais diversas. O conhecimento sobre o uso e os efeitos das drogas auxilia no enfrentamento da complexidade deste fenômeno, contribuindo em programas de prevenção, tratamento, reabilitação e reinserção dos usuários em vários segmentos da sociedade. Assim, o uso de drogas e seus efeitos são considerados graves problemas sociais e de saúde pública mundial, pois consistem em fatores de risco para o acidente/trauma e/ou a violência(1-2).

Ocasiona intercorrências indesejáveis, como crises familiares, atos violentos e internações hospitalares, com agravos que geram elevados custos sociais e financeiros, despertando a atenção do poder público e dos profissionais da saúde, com casos que podem culminar em mortes e perdas funcionais temporárias e permanentes ${ }^{(3-4)}$.

No âmbito mundial, o uso abusivo do álcool é responsável por 5,9\% das mortes e não se observa tendência à diminuição e nem à estabilização de casos. A Organização Mundial da Saúde (OMS) identificou que o álcool está entre os 20 maiores fatores de risco para doenças crônicas não transmissíveis e, quando somado às drogas ilícitas, a prevalência anual ultrapassa 200 milhões de casos de doenças ${ }^{(5)}$.

Usuários de drogas de abuso são hospitalizados pelos efeitos clínicos causados pelo uso agudo ou binge (consumo esporádico e excessivo em curto período de tempo)(6), em decorrência de alterações fisiopatológicas secundárias ao uso prolongado ou crônico destas substâncias ou por associação a violências e traumas, considerados também efeitos secundários ${ }^{(7)}$.

Os efeitos nocivos no organismo, decorrentes do uso nocivo de drogas por longo período, exigem cuidados especializados, devido à gravidade dos casos, aumentando o número de internações destes pacientes em unidade de terapia intensiva, tendo, como consequência, maior número de óbitos e/ou sequelas ao indivíduo(8).

As unidades de terapia intensiva são ambientes onde os pacientes se encontram em grave estado clínico, com comprometimento das funções vitais e risco de morte. Nestas unidades, pacientes que fazem uso de drogas de abuso são internados, principalmente, por complicações clínicas ou traumáticas, agudas ou crônicas agudizadas, geralmente relacionadas com a gravidade da dependência à droga ${ }^{(8)}$.

Embora se considere importante caracterizar este subgrupo populacional para a construção de estratégias de prevenção e controle dos agravos associados ao uso e ao abuso de substâncias psicoativas, há poucos estudos na literatura nacional sobre internações de pacientes com intoxicação por abuso de drogas em unidades de terapia intensiva.
O objetivo deste estudo foi caracterizar o perfil epidemiológico de internações em terapia intensiva decorrentes de efeitos secundários do uso de álcool e outras drogas de abuso.

\section{Método}

Estudo seccional, do tipo transversal e quantitativo, das internações em unidade de terapia intensiva adulto de um hospital escola, localizado na região noroeste do Estado do Paraná, no período de janeiro de 2011 a dezembro de 2015, notificadas ao Centro de Controle de Intoxicações e com diagnósticos médicos ou complicações relacionadas ao consumo de drogas de abuso. Os dados foram obtidos das fichas epidemiológicas de Ocorrência Toxicológica/Intoxicação Alcoólica e/ou outras Drogas.

Os critérios de inclusão foram pacientes de ambos os sexos, com diagnósticos médicos associados a efeitos nocivos ao uso/abuso de drogas, internados na unidade de terapia intensiva adulto de um hospital universitário e notificados ao Centro de Controle de Intoxicações do Hospital Regional Universitário de Maringá, um serviço de apoio às urgências toxicológicas, atendendo em regime de plantão de 24 horas.

O hospital em estudo é credenciado ao Sistema Único de Saúde (SUS), caracterizando-se como instituição de caráter público, e prioriza atividades de assistência, ensino e pesquisa. A clientela compreende a população dos municípios da região noroeste do Estado, com 686.471 habitantes. A unidade de terapia intensiva adulto constitui-se de oito leitos, sendo referência de leitos públicos para atendimento a pacientes graves da região. Atende, ainda, pacientes clínicos e cirúrgicos, de ambos os sexos e com idade mínima de 13 anos.

Notificaram-se, utilizando a categoria de intoxicação por álcool ou outras drogas de abuso, 2.166 casos ao Centro de Controle de Intoxicações no período em estudo. Destas notificações, 584 (26,9\%) foram de internações hospitalares e, destas, 138 (23,6\%) necessitaram de internação em unidade de terapia intensiva. Neste sentido, a população em estudo foi composta por 138 pacientes em estado grave.

Como fontes de dados, foram utilizadas as fichas de Ocorrência Toxicológica e de Intoxicação Alcoólica e/ ou outras Drogas arquivadas no Centro de Controle de Intoxicações. As variáveis estudadas compreenderam características dos eventos, tendo sido compilados os dados relacionados à data da internação: ano em que foi gerada a notificação dos casos, organizados e apresentados no período de 2011 a 2015; dados sociodemográficos; dados da intoxicação; circunstância da intoxicação; dados da internação e do tipo de tratamento; comorbidades; tempo de internação em dias de internação em unidade de terapia intensiva adulto e desfecho clínico do caso. 
Os dados coletados foram compilados em uma planilha do Microsoft Exce ${ }^{\circledR} 2013$ e analisados com o auxílio do software Statistical Pakage for Social Science $(\text { SPSS })^{\circledR}$, versão 24. A fim de avaliar associações significativas entre as variáveis sociodemográficas e clínicas, foram utilizadas a estatística descritiva, por meio de medidas de tendência central, e a estatística univariada, com uso do qui-quadrado, teste exato de Fischer, sendo consideradas significativas aquelas com $\mathrm{p}<0,05$ em intervalo de confiança de 95\%.

\section{Resultados}

No período do estudo, foram analisadas 138 fichas com registro de Ocorrência Toxicológica/Intoxicação Alcoólica de internações de pacientes na unidade de terapia intensiva adulto que se enquadraram nos critérios de inclusão. A média anual de internações foi 27,6 ao ano, variando de 12 a 36 casos ao ano.

As características sociodemográficas estão apresentadas na Tabela 1. A idade dos pacientes internados em terapia intensiva variou de 13 a 86 anos, com média de idade de 47,9 anos (DP $\pm 16,4$ ). Destaca-se um grande número de aposentados devido à idade da amostra da pesquisa.

Ainda em relação à idade, a maioria dos casos $(66,7 \%)$ ocorreu com pessoas com mais de 40 anos, e esta proporção esteve associada ao tipo de agente tóxico com maior prevalência no estudo, que foi o uso de álcool e/ou álcool associado a outras drogas. Verificou-se associação estatística significativa entre a idade dos indivíduos e ocorrências e óbitos.

Tabela 1 - Características sociodemográficas de 138 pacientes internados em terapia intensiva decorrente de efeitos secundários do uso de álcool e outras drogas de abuso, no período de 2011 a 2015. Maringá, PR, Brasil, 2018

\begin{tabular}{lc}
\hline \multicolumn{1}{c}{ Variáveis } & Resultados $\mathbf{n}(\%)$ \\
\hline Sexo & $123(89,13)$ \\
Masculino & $15(10,87)$ \\
Feminino & \\
Idade & 3,62 \\
Menor 18 anos & 16,66 \\
19 a 34 anos & 34,78 \\
35 a 50 anos & 30,43 \\
51 a 64 anos & 14,49 \\
Acima 65 anos & \\
Escolaridade (n=88) & $2(2,27)$ \\
Analfabeto & $54(61,38)$ \\
Ensino Fundamental Incompleto & $10(11,36)$ \\
Ensino Fundamental Completo & $10(11,36)$ \\
Ensino Médio Incompleto & $10(11,36)$ \\
Ensino Médio Completo & $2(2,27)$ \\
Ensino Superior & \\
Profissão (n=94) & $48(51,07)$ \\
Vínculo Empregatício & $25(26,59)$ \\
Sem Vínculo Empregatício & $21(22,34)$ \\
Aposentado &
\end{tabular}

Em relação ao sexo dos indivíduos, 89,1\% eram do sexo masculino. Foi verificada razão de 8,4 homens para cada mulher acometida. Apesar de a maioria da amostra ser do sexo masculino e a maioria dos óbitos ter ocorrido nestes indivíduos, não foi verificada associação estatística significativa no teste qui-quadrado.

Observou-se que $43,5 \%$ tinham até oito anos de escolaridade, $20,3 \%$, mais de oito anos de escolaridade, e, em um grande número de casos, esta informação foi negligenciada ou não informada $(36,2 \%)$. Não foi possível associar estatisticamente a ocorrência de óbito e a escolaridades dos pacientes internados.

Os pacientes internados na unidade de terapia intensiva adulto eram provenientes, principalmente, do pronto-socorro do hospital $(85,5 \%) ; 8,7 \%$ dos indivíduos eram provenientes da clínica cirúrgica e $5,8 \%$, da clínica médica. Não foi observada associação estatística significativa entre o setor proveniente e a ocorrência de óbitos.

No que se refere às condições ocupacionais e de empregabilidade dos indivíduos internados na unidade de terapia intensiva adulto, observou-se uma gama diversa de profissões, o que não permitiu associações estatísticas significativas, porém, $14,5 \%$ dos internados trabalhavam na construção civil. Outra característica da amostra foi que 13,7\% eram aposentados e, em 31,9\%, a profissão foi ignorada.

Os dados relacionados às drogas de abuso que ocasionaram os agravos que resultaram em internação em UTI estão descritos na Tabela 2. Observou-se que a droga mais relacionada à internação foi o álcool, presente em $92,7 \%$ dos diagnósticos médicos registrados. Sem associação com outra droga, o álcool foi responsável pela internação de 116 (84,0\%) pacientes e, com associação a outras drogas ilícitas (crack, cocaína e maconha), com $12(8,6 \%)$. O crack foi responsável por seis $(4,3 \%)$ casos, a maconha, por dois $(1,4 \%)$ casos e cocaína, por dois $(1,4 \%)$ casos. O diagnóstico de intoxicação por drogas foi baseado em dados registrados pela equipe médica e de Enfermagem no prontuário médico hospitalar.

Com relação às drogas de abuso consumidas pelos homens internados na unidade de terapia intensiva adulto, $98,5 \%$ eram usuários de bebida alcoólica, sendo $16 \%$ homens usuários de álcool associado a outras drogas de abuso. Apenas $1,5 \%$ era usuário exclusivo de outras drogas.

Tabela 2 - Distribuição de frequências de pacientes internados em UTI notificados ao CCI/HUM por internação em decorrência de consumo de drogas de abuso. Maringá, PR, Brasil, 2018

\begin{tabular}{lc}
\hline \multicolumn{1}{c}{ Droga de abuso } & $\mathbf{n}(\%)$ \\
\hline Álcool & $116(84)$ \\
Álcool e outras drogas & $12(8,6)$ \\
Crack & $6(4,3)$ \\
Maconha & $2(1,4)$ \\
Cocaína & $2(1,4)$ \\
\hline
\end{tabular}


O abuso crônico das drogas foi a circunstância da intoxicação predominante em 97,1\% dos casos, mesmo naqueles associados ao trauma. Com relação à etiologia das comorbidades nos pacientes hospitalizados na unidade de terapia intensiva adulto, 97,1\% eram relacionadas às condições crônicas agudizadas.

O cruzamento entre as variáveis sociodemográficas e a ocorrência de óbitos entre os pacientes internados está sumarizado na Tabela 3.

Com relação aos diagnósticos médicos de internação, codificados pela Classificação Estatística Internacional de Doenças e Problemas Relacionados com a Saúde - 10a edição (CID10), 2,2\% tinham diagnóstico de doenças endócrinas, nutricionais e metabólicas; $21 \%$ receberam diagnóstico de transtornos mentais e comportamentais; $12,3 \%$ dos internados receberam diagnóstico de doenças do sistema nervoso; 5,1\% foram diagnosticados com doenças do aparelho circulatório; $12,3 \%$, com doenças do aparelho respiratório; $32,6 \%$, com doenças do aparelho digestivo, sendo este o diagnóstico com maior número de óbitos. Três pacientes $(2,2 \%)$ receberam códigos para propósitos especiais (síndrome respiratória aguda grave) e 12,3\%, diagnósticos de causas externas de morbidade e de mortalidade. Houve associação estatística significativa entre os diagnósticos médicos e a ocorrência de óbitos.

A duração da internação hospitalar variou de um a 256 dias. A média foi 16,6 dias (desvio-padrão $\pm 24,5$ dias), a mediana foi dez dias e a moda, de três dias. Verificou-se associação estatística significativa entre a duração da internação e a ocorrência de óbitos, indicando risco maior para aquelas internações que foram abaixo da média de 16,6 dias. A taxa de ocupação de leitos da unidade de terapia intensiva adulto por usuários de álcool e outras drogas, no período estudado, foi de $15,6 \%$ ao ano.

Avaliou-se a abordagem clínica administrada aos pacientes, observando-se que, em $66,7 \%$ dos casos, os pacientes receberam tratamento clínico e, em 33,3\%, eles receberam tratamentos traumatológicos e/ou cirúrgico. Não foi observada, na amostra, associação significativa entre o tipo de tratamento e os óbitos.

Como desfecho da internação na unidade de terapia intensiva adulto, $61,6 \%$ dos pacientes receberam alta hospitalar (entre dois a 89 dias de internação em unidade de terapia intensiva, com a média de 15,78 dias para a alta hospitalar), e 38,4\% evoluíram para óbito durante a internação (entre um a 230 dias de internação em unidade de terapia intensiva, com média de 19 dias para o óbito).

Tabela 3 - Referência cruzada entre variáveis sociodemográficas e ocorrência de óbitos em 138 pacientes internados em unidade de terapia intensiva adulto por efeitos associados a drogas de abuso. Maringá, PR, Brasil, 2018

\begin{tabular}{|c|c|c|c|c|}
\hline Internados em UTI* & n (\%) & Alta (n) & Óbitos (n) & Valor de $p$ \\
\hline \multicolumn{5}{|l|}{ Idade, anos } \\
\hline Até 40 & $46(33,3)$ & 35 & 11 & \multirow{2}{*}{$0,013 \dagger$} \\
\hline$>40$ & $92(66,7)$ & 50 & 42 & \\
\hline \multicolumn{5}{|l|}{ Sexo } \\
\hline Masculino & $123(89,1)$ & 77 & 46 & \multirow{2}{*}{0,486} \\
\hline Feminino & $15(10,9)$ & 8 & 7 & \\
\hline \multicolumn{5}{|l|}{ Escolaridade, anos } \\
\hline Até 8 & $60(43,5)$ & 40 & 20 & \multirow{3}{*}{0,098} \\
\hline$>8$ & $28(20,3)$ & 20 & 08 & \\
\hline Ignorada & $50(36,2)$ & 25 & 25 & \\
\hline \multicolumn{5}{|l|}{ Setor de origem } \\
\hline Centro cirúrgico & $12(8,7)$ & 7 & 5 & \multirow{3}{*}{0,475} \\
\hline Clínica médica & $8(5,8)$ & 7 & 1 & \\
\hline Pronto-socorro & $118(85,5)$ & 71 & 47 & \\
\hline \multicolumn{5}{|l|}{ Uso no tempo } \\
\hline Crônico & $134(97,1)$ & 82 & 52 & \multirow{2}{*}{0,502} \\
\hline Agudo & $4(2,9)$ & 3 & 1 & \\
\hline \multicolumn{5}{|l|}{$C I D^{\ddagger} 10$} \\
\hline E00-E99 & $3(2,2)$ & 0 & 3 & \multirow{8}{*}{$<0,001^{\S}$} \\
\hline F00-F99 & $29(21)$ & 23 & 6 & \\
\hline G00-G99 & $17(12,3)$ & 14 & 3 & \\
\hline 100-199 & $7(5,1)$ & 6 & 1 & \\
\hline J00-J99 & $17(12,3)$ & 12 & 5 & \\
\hline K00-K93 & $45(32,6)$ & 16 & 29 & \\
\hline U00-U99 & $3(2,2)$ & 3 & 0 & \\
\hline V01-Y98 & $17(12,3)$ & 11 & 6 & \\
\hline \multicolumn{5}{|l|}{ Tempo de internação } \\
\hline Até 17 dias & $64(46,4)$ & 32 & 32 & \multirow{2}{*}{$0,009^{\dagger}$} \\
\hline$>17$ dias & $74(53,6)$ & 53 & 21 & \\
\hline \multicolumn{5}{|l|}{ Abordagem terapêutica } \\
\hline Clínica & $92(66,7)$ & 53 & 39 & \multirow{2}{*}{0,119} \\
\hline Cirurgia/trauma & $46(33,3)$ & 32 & 14 & \\
\hline
\end{tabular}




\section{Discussão}

Os limites dos resultados deste estudo estão relacionados à utilização de dados coletados de fontes indiretas, ou seja, fichas de notificação de ocorrências toxicológicas, o que pode resultar em dados subnotificados ou incompletude, como foi verificado em algumas variáveis. Por outro lado, esta utilização apresenta vantagens, uma vez que, dentro das possibilidades da obtenção dos dados, permite conhecer comorbidades e aspectos dos pacientes que podem subsidiar protocolos clínicos (médicos e de Enfermagem), bem como desvelar, ao poder público, dados que podem resultar em políticas públicas de prevenção e redução de danos.

O perfil etário dos indivíduos demonstra que a maioria era de adultos jovens em idade economicamente ativa. Destaca-se o grande número de aposentados devido ao perfil etário da amostra. Tais achados permitem supor que os agravos à saúde e as incapacidades, gerados pelo uso problemático das drogas, são importantes, como identificado em estudos anteriores, nos quais se verificou que o uso de álcool aumenta a vulnerabilidade dos idosos e adultos jovens para quedas, lesões e, também, quanto às interações álcool-droga ${ }^{(8-9)}$.

O maior número de casos de internação ocorreu em idades acima de 35 anos. Esta proporção está associada ao tipo de agente tóxico mais prevalente no estudo: o álcool e o álcool associado a outras drogas. Tal padrão é também observado em outros países, como na Rússia, onde o uso indevido de álcool é responsável por mais de metade das mortes entre os homens em idade economicamente ativa. Pontua-se que os principais motivos para este padrão de consumo são a pobreza e a falta de satisfação "espiritual e cultural"; como motivos secundários, encontram-se o preço, a acessibilidade e a popularidade das bebidas alcoólicas ${ }^{(10)}$.

O estudo confirma a maior prevalência de internações e de mortes por alcoolismo em indivíduos do sexo masculino, os quais apresentam maior exposição e, consequentemente, efeitos graves do uso das drogas ao organismo. Esta tendência de consumo não é diferente de outras regiões do país, nas quais a prevalência de óbitos e dos usuários dependentes de drogas de abuso, em especial, o álcool, dá-se entre a população do sexo masculino ${ }^{(8,10-11)}$.

A baixa escolaridade encontrada entre os pacientes corrobora outros estudos que verificaram que o status socioeconômico é um dos muitos fatores que influenciam o consumo de álcool de uma pessoa e os resultados relacionados a este consumo. A dependência de álcool e de outras drogas é uma amalgamação complexa, de múltiplos fatores, como raça, etnia, status sociodemográfico, status educacional e sexo. Entre os indivíduos com baixo status sociodemográfico, ou seja, em um cenário de vulnerabilidade, experimentam-se maiores consequências relacionadas ao consumo de álcool(12-13).

Em estudo sobre o risco relacionado ao consumo de drogas em trabalhadores da construção de uma cidade da região Sul do Brasil, verificou-se que o álcool, o tabaco, a maconha e a cocaína são as principais substâncias utilizadas por estes trabalhadores. 0 desemprego também foi destaque na pesquisa, estando associado à dependência do uso de drogas e às baixas aceitação e adesão ao tratamento, além da reinserção social destes usuários ${ }^{(14)}$.

O álcool, uma droga lícita, foi o responsável pela maioria das internações, isoladamente ou associado a outras drogas ilícitas, relacionando-se ao uso crônico da droga e ao período de longa internação. Por seu turno, as intoxicações alcoólicas agudas são responsáveis pela maior incidência em jovens, com aumento da gravidade dos casos, com grau máximo de alteração da consciência provocada pelo consumo em excesso, coma, sequelas temporais e permanentes, inclusive, a morte. Estes dados corroboram outro estudo que discute o fato de o álcool ser o principal protagonista das doenças traumáticas da atualidade ${ }^{(15)}$.

O álcool é um potencializador dos efeitos de outras drogas, quando utilizadas concomitantemente, pois gera maior toxicidade e prolonga os efeitos. Geralmente, internações por drogas de abuso estão relacionadas às complicações do consumo compulsivo com comprometimento clínico devido ao uso crônico ou a situações de violência e trauma. Os danos provocados, direta ou indiretamente, pelo consumo do álcool, resultam na presença de comorbidades ${ }^{(2,16)}$.

No caso das intoxicações agudas de drogas de abuso, existe relação direta com as causas externas: acidentes, quedas e violências. As doenças mentais também estão associadas à dependência de substâncias psicoativas e têm um aumento significativo nos últimos anos. Transtornos mentais, como a esquizofrenia, transtorno afetivo bipolar e depressão, são os mais associados ao uso de drogas, tendo como agravante a tentativa de suicídio(17).

Ao comparar as internações hospitalares por drogas de abuso no Centro de Controle de Intoxicações nos anos estudados, ocorreram $23,6 \%$ de internações em unidade de terapia intensiva. Isso demonstra os reflexos do aumento do consumo de substâncias psicoativas na população e gera agravos à saúde física e mental da população, e, consequentemente, gravidade clínica associada ao abuso de drogas, o que, por sua vez, gera alterações fisiopatológicas, necessitando de cuidados intensivos.

A Organização Mundial da Saúde aponta que o risco de danos está associado a uma série de características individuais, como idade, sexo, predisposição, padrão de 
consumo (agudo e crônico) e fatores sociodemográficos. Os problemas relacionados ao abuso de álcool não se restringem exclusivamente aos indivíduos dependentes, estando associados a padrões de uso. As complicações em decorrência do abuso crônico, muitas vezes, geram implicações severas, danos irreversíveis e a morte(18).

A exposição crônica ao álcool pode acarretar um nível de toxicidade que atinge, direta ou indiretamente, importantes órgãos ou sistemas corporais e, consequentemente, origina doenças como a cirrose hepática, doença comum encontrada nesta população. Estudos apontam o álcool como a droga mais consumida no Brasil, sendo que o etilismo atinge de cinco a $10 \%$ da população adulta brasileira(18-19).

A população brasileira está entre as maiores consumidoras de bebida alcoólica e as taxas de utilização continuam em crescimento. A prevalência do consumo abusivo de álcool na população brasileira foi de $13,7 \%$ e a proporção entre homens foi 3,3 vezes maior do que entre as mulheres. O álcool tem permanecido como uma prioridade relativamente baixa nas políticas públicas, incluindo as de saúde, apesar das elevadas cargas social, sanitária e econômica associadas ao consumo nocivo do mesmo(19).

Na população estudada, os pacientes encontravam-se em situação de extrema gravidade, mostrando que os efeitos deletérios do consumo de uma droga, seja agudo ou crônico, realmente podem interromper a vida do indivíduo. Podem, também, ocasionar sequelas físicas e/ou emocionais temporárias e permanentes, que se estendem à família e à sociedade. O tempo de internação está ligado diretamente à gravidade clínica dos casos e o desfecho, em $38,4 \%$ dos casos, foi o óbito, o que comprova a indicação de internação em terapia intensiva( ${ }^{(8)}$.

Não se pode deixar de ressaltar que existe subnotificação pelas equipes nos serviços de saúde no que diz respeito aos atendimentos e internações nos casos em que o paciente fez uso de drogas de abuso. Essa vigilância é deficiente nos hospitais em geral, principalmente naqueles onde não existem centros de assistência toxicológica, com maior escassez de dados que possam ser confrontados com a situação clínica dos pacientes. Outro fator a se considerar é a gravidade dos pacientes, que dificulta a obtenção destes $\operatorname{dados}^{(8)}$.

As pesquisas relacionadas às drogas de abuso, gravidade clínica e internação em terapia intensiva contribuem para a construção do conhecimento da Enfermagem relacionado aos cuidados com usuário de drogas em situações de risco de morte. O enfermeiro, como promotor do cuidado, deve atuar junto da equipe, do doente e dos familiares, colocando em prática ações e intervenções do cuidado ao paciente grave e desenvolvendo estratégias de prevenção junto de familiares para a reabilitação e reinserção social do doente após a alta hospitalar.

\section{Conclusão}

O perfil de prevalência apresentado é importante, por revelar grupos vulneráveis entre os usuários de álcool e outras drogas, demonstrando que homens adultos jovens, em idade economicamente ativa, com uso crônico de álcool e com comorbidades evoluem para óbito, mesmo com tratamento em unidade de terapia intensiva.

\section{Agradecimentos}

À Erica Gomes de Almeida pela colaboração no processo de coleta de dados.

\section{Referências}

1. Sudhinaraset M, Wigglesworth C, Takeuchi DT. Social and Cultural Contexts of Alcohol Use. Influences in a Social-Ecological Framework. Alcohol Res. 2016; 38(1):35-45.

2. Santana CJ, Oliveira ML. [Effects of drug involvement on long-term users' family members]. Rev Rene. 2017; 18(5):671-8. doi: 10.15253/21756783.2017000500015. Portuguese.

3. Korcha RA, Cherpitel CJ, Witbrodt J, Borges G, HejaziBazargan S, Bond JC, et al. Violence-related injury and gender: The role of alcohol and alcohol combined with illicit drugs. Drug Alcohol Rev. 2014; 33(1):43-50. doi: 10.1111/dar.12087

4. Okumura Y, Shimizu S, Ishikawa KB, Matsuda S, Fushimi K, Ito $\mathrm{H}$. Comparison of emergency hospital admissions for drug poisoning and major diseases: a retrospective observational study using a nationwide administrative discharge database. BMJ Open. 2012; 2(6):pii: e001857. doi: 10.1136/bmjopen-2012-001857 5. Pereira GB, Oliveira MM. [Hospitalizations in intensive care units related to the abuse of alcohol, tobacco and illicit drug]. VITTALLE. 2016; 28(1):49-64. Portuguese. 6. West RK, Maynard ME, Leasure JL. Binge ethanol effects on prefrontal cortex neurons, spatial working memory and task-induced neuronal activation in male and female rats. Physiol Behav. 2018; 188:79-85. doi: 10.1016/j.physbeh.2018.01.027.

7. Rocha GS. Fatores associados, gravidade do trauma e sequelas de acidentes de transporte terrestre: um estudo a partir de egressos hospitalares. São Paulo: USP; 2015.

8. Antunes F, Oliveira ML. [Characteristics of patients hospitalized at an ICU due to drug abuse]. Investig y Educ Enferm. 2013; 31(2):201-9. Portuguese.

9. Zolot J. Age, Racial, and Gender Gaps Are Narrowing in Alcohol Use Disorders. Am J Nurs. 2017; 117(11):14. doi: 10.1097/01.NAJ.0000526731.98527.dc

10. Jang HS, Kim JY, Choi SH, Yoon YH, Moon SW, Hong YS, et al. Comparative analysis of acute toxic poisoning 
in 2003 and 2011: Analysis of 3 academic hospitals. J Korean Med Sci. 2013; 28(10):1424-30. doi: 10.3346/ jkms.2013.28.10.1424

11. Malta DC, Bernal RT, Mascarenhas MD, Silva MM, Szwarcwald $\mathrm{CL}$, Morais Neto $\mathrm{OL}$, et al. [Alcohol consumption and driving in Brazilian capitals and Federal District according to two national health surveys]. Rev Bras Epidemiol. 2015; 18(suppl 2):214-23. doi: http://dx.doi. org/10.1590/1980-5497201500060019. Portuguese.

12. Organização Internacional do trabalho (OIT). Perfil do trabalho docente no Brasil: um olhar sobre as unidades da federação [Internet]. Brasilia, DF: OIT; 2012 [Acesso 22 ago 2018]. Disponível em: : http://www.ilo. org/wcmsp5/groups/public/---dgreports/---integration/ documents/publication/wcms_206109.pdf

13. Gavioli A, Mathias TA, Rossi RM, Oliveira ML. Risks related to drug use among male construction workers. Acta Paul Enferm. 2014; 27(5):471-8. doi: http://dx.doi. org/10.1590/1982-0194201400077

14. Silva BD, Santos JD, Santos AM, Madeira MZ, Gouveia MT. Acidentes com motocicletas: características da ocorrência e suspeita do uso de álcool. Cogitare Enferm. 2017; 22(3):50715. doi: http://dx.doi.org/ 10.5380/ce.v22i3.50715

15. Mangueira SO, Guimarães FJ, Mangueira JO, Fernandes AF, Lopes MV, Mangueira SO, et al. [Health promotion and public policies of alcohol in Brazil: integrative literature review]. Psicol Soc. 2015; 27(1):157-68. doi: http://dx.doi.org/10.1590/180703102015v27n1p157.Portuguese.

16. Silva ER, Ferreira AC, Borba LO, Kalinke LP, Nimtz MA, Maftum MA. [Drug use impact in drug addicts' physical and mental health]. Ciênc Cuidado Saúde. 2016;15(1):101-8. doi: 10.4025/cienccuidsaude. v15i1.27137. Portuguese.

17. World Health Organization (WHO). Global status report on alcohol and health 2014. Geneve, Switzerland: WHO; 2014.

18. Garcia LP, Freitas LR. Heavy drinking in Brazil: results from the 2013 National Health Survey. Epidemiol Serviços Saúde. 2015; 24(2):227-37. doi: http://dx.doi.org/ 10.5123/S1679-49742015000200005.

\section{Contribuição dos autores}

Concepção e desenho da pesquisa: Cleiton José Santana, Anai Adário Hungaro, Magda Lúcia Félix de Oliveira. Obtenção de dados: Cleiton José Santana, Anai Adário Hungaro, Rosangela Cristophoro, Indianathan de kassia Santana Elvira. Análise e interpretação dos dados: Cleiton José Santana, Anai Adário Hungaro, Rosangela Cristophoro, Indianathan de kassia Santana Elvira, Magda Lúcia Félix de Oliveira. Análise estatística: Aroldo Gavioli. Redação do manuscrito e Revisão crítica do manuscrito quanto ao conteúdo intelectual importante: Cleiton José Santana, Anai Adário Hungaro, Rosangela Cristophoro, Indianathan de kassia Santana Elvira, Aroldo Gavioli, Magda Lúcia Félix de Oliveira. Orientação: Magda Lúcia Félix de Oliveira.

Todos os autores aprovaram a versão final do texto.

Conflito de interesse: os autores declararam que não há conflito de interesse. 